\title{
Clubfoot Correction in Walking-age Children: A Review
}

\author{
Norgrove Penny ${ }^{1}$, Alaric Aroojis ${ }^{2}$, Anil Mehtani ${ }^{3}$, Bibek Banskota ${ }^{4}$
}

\begin{abstract}
Children with clubfoot often present after the walking age, especially in low- and middle-income countries where approximately $80 \%$ of children with clubfoot are born. With advancing age, there is increased stiffness of the soft tissue structures and decreased remodeling potential of the bones of the foot. Not all clubfeet in older children are rigid-some are flexible and amenable to stretching and conservative treatment. Hence, the initial evaluation of the deformity must include an assessment of correctability. The treatment of clubfoot in the older child is challenging and was traditionally performed using complex soft tissue and bony surgeries, often with poor outcomes in the long term. Recent literature has focused on the role of conservative treatment utilizing Ponseti principles of serial manipulation and casting, combined with limited surgery. The purpose of this review is to report the changing trends in the management of clubfoot in the walking child, to review the current literature regarding various treatment modalities, and to recommend a practical approach to treatment based upon age, inherent flexibility of the foot, available resources, and contextual factors.
\end{abstract}

Keywords: Clubfoot, Fixator, Osteotomy, Ponseti, Surgery, Walking age.

Journal of Foot and Ankle Surgery (Asia Pacific) (2021): 10.5005/jp-journals-10040-1166

\section{Burden of Clubfoot Disability in Countries With Limited Resources}

Clubfoot is the most common congenital musculoskeletal deformity leading to locomotor impairment. Practitioners in low- and middle-income countries (LMICs) are often faced with children presenting in all age groups after walking age, including adulthood. Owing to limited medical resources, lack of trained healthcare providers, fragile healthcare systems, and a host of other challenges like poverty and illiteracy, clubfoot treatment in LMICs is often delayed or inadequate. The problem is not just restricted to LMICs. Developed countries, too, have populations who experience insurmountable barriers in accessing care, resulting in children presenting after walking age. This is particularly prevalent among immigrants of refugee populations. The purpose of this review is to focus on children presenting with untreated or residual clubfoot deformity after walking age.

\section{Pathoanatomy of Clubfoot and the Older CHILD}

The act of walking imposes unique challenges upon a clubfoot deformity due to increasing stiffness of the contracted soft tissues and advancing maturation, ossification, and pathologic remodeling of the osteoarticular structures of the foot. The connective tissues of a newborn are readily stretchable, and the cartilaginous bones readily remodel as treatment is instituted. This has been confirmed elegantly in a previous MRI study. ${ }^{1}$ As a child grows older, remodeling potential diminishes. The question is when such remodeling no longer occurs. Another MRI study has shown remodeling of the bone structure in a 7-year-old. ${ }^{2}$ Significant bone maturity will likely have occurred by 10 years of age and bone remodeling would be predicted to be less readily accomplished in older age groups, the shape of the bones encouraging relapse to the original position after casting. The anatomic changes in the bone shape seen in the dissections of Ponseti or the MRI studies \begin{tabular}{l}
\hline 1Branch for International Surgical Care, University of British Columbia, \\
Victoria, British Columbia, Canada \\
${ }^{2}$ Department of Paediatric Orthopaedics, Bai Jerbai Wadia Hospital for \\
Children, Mumbai, Maharashtra, India \\
${ }^{3}$ Department of Orthopaedics, Lady Hardinge Medical College, New \\
Delhi, India \\
${ }^{4}$ Department of Orthopedic, B and B Hospital, Lalitpur, Kathmandu, \\
Nepal \\
Corresponding Author: Alaric Aroojis, Department of Paediatric \\
Orthopaedics, Bai Jerbai Wadia Hospital for Children, Mumbai, \\
Maharashtra, India, Phone: +91 9320284402, e-mail: aaroojis@gmail. \\
com \\
How to cite this article: Penny N, Aroojis A, Mehtani A, et al. Clubfoot \\
Correction in Walking-age Children: A Review. J Foot Ankle Surg (Asia \\
Pacific) 2021;8(3):102-109. \\
Source of support: Nil \\
Conflict of interest: None \\
\hline \hline
\end{tabular}

of Pirani persist in the child who is not treated before walking age. It is also recognized clinically that children presenting in walking age with untreated clubfoot deformity have variable degrees of flexibility and deformity.

Not all clubfeet in older children are the same. Penny ${ }^{3}$ has described two generic types of clubfoot deformity in older children, depending on the degree of fixed cavus and the flexibility of the foot (Fig. 1). Where cavus is marked, children walk on the dorsum of the foot with the toes facing backward. Where cavus is not as prevalent and where there is intrinsic flexibility remaining in the midfoot children walk on the side of the foot with the toes facing more or less forward. This basic clinical assessment is useful in predicting the efficacy of casting; the former is more rigid and more recalcitrant to serial manipulation and casting while the latter is more likely to respond to conservative treatment due to its inherent flexibility.

(0) The Author(s). 2021 Open Access This article is distributed under the terms of the Creative Commons Attribution 4.0 International License (https:// creativecommons.org/licenses/by-nc/4.0/), which permits unrestricted use, distribution, and non-commercial reproduction in any medium, provided you give appropriate credit to the original author(s) and the source, provide a link to the Creative Commons license, and indicate if changes were made. The Creative Commons Public Domain Dedication waiver (http://creativecommons.org/publicdomain/zero/1.0/) applies to the data made available in this article, unless otherwise stated. 


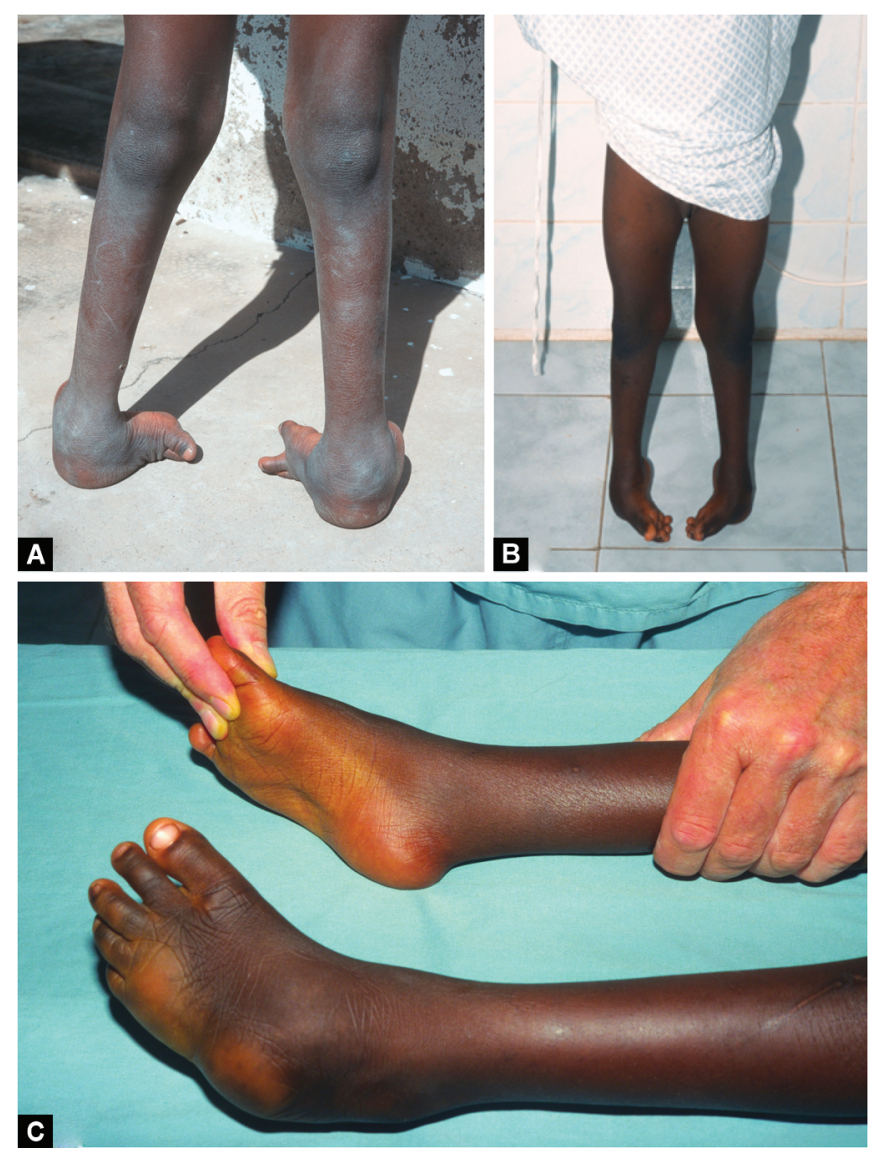

Figs 1 A to C: (A) A 7-year-old child with untreated clubfoot with marked cavus and toes pointing backward indicating a very stiff foot. (B) A 6-year-old child with untreated clubfoot walking on the side of the foot and $(C)$ showing significant flexibility of the midfoot

\section{Evaluation of the Deformity in Older ChILDREN}

The scoring method of Pirani has become widely utilized, given its validation clinically and with MRI studies and its simplicity of use. It is validated in babies but not in older children. The more extensive Dimeglio score may be more applicable to older children. A scoring system has been developed in Ethiopia specifically for the LMIC environment focusing on older children. ${ }^{4}$ This utilizes the measurement of plantaris, adduction, varus, equinus, and rotation, giving the acronym PAVER to the score. This score has been validated against the Pirani and Dimeglio scores and includes multipliers according to age. The score is practical to apply in the clinical environment and can predict resistance to casting treatment. Using the multipliers allows for the adaptation of the treatment programs depending on the age of presentation. The PBS score has also been recently introduced as a clinical assessment tool for walking-age children, scoring functional outcomes after treatment and identifying early signs of relapse. ${ }^{5}$ The seven-item score assesses important functional components while standing and walking (hindfoot varus, standing supination, walking supination, and early heel rise) in addition to passive and active ankle dorsiflexion and subtalar abduction while sitting.

\section{Traditional Treatment Algorithm}

The traditional treatment of untreated clubfoot in the walking age older child was considered to be primarily surgical. Penny ${ }^{3}$ reported an algorithmic approach to the surgical treatment of clubfoot deformity in the older child, progressing from soft tissue surgeries alone through soft tissue releases combined with osteotomies to isolated osteotomies or arthrodesis. He emphasized that age is not necessarily a predictor of the type of surgery required; severity of deformity and inherent flexibility are more important. The canny surgeon would have access to a full range of different surgeries in their armamentarium. However, during the last decade, there has been a surge of interest in extending the use of the Ponseti method to older children as well.

\section{Changing Trends and Role of the Ponseti Method in the Older Child}

Dr Ponseti developed his technique of serial manipulation and casting for newborns presenting at $<6$ months of age. Initial uptake of the technique around the world was felt to be applicable in babies, with standard surgical techniques necessary in older children. Early experience in Brazil and Nepal, however, proved that the technique could be applied effectively to older children as well. ${ }^{6-8}$ What then is the upper limit for the use of the technique and what is the role of adjuvant surgical procedures?

The success of the Ponseti manipulative technique depends on the pliability of the soft tissues and the ability of the tarsal bones to remodel. Is there a point in childhood where these two processes limit the efficacy of manipulation and percutaneous Achilles tenotomy? How does the technique described by Ponseti need to be adapted or modified to treat walking-age children? Numerous studies have now been published showing the efficacy of Ponseti principles of casting in older children, borne out by the experience of practitioners in high-volume clinics, particularly in Asia. Few studies, however, discriminate based on age. The correctability of foot deformity in an 18-month-old is presumably very different from correctability in a 10- or 18-year-old.

A study in Nepal of children in the 1-6-year-old age group showed initial correction could be achieved without extensive soft tissue release in $94 \% .{ }^{8} \mathrm{~A}$ long-term study of this group of patients, followed for at least 10 years, demonstrated that success was maintained over the long run, with a plantigrade foot achieved in 95\%. Patient-reported outcomes were favorable, although residual deformities were common. ${ }^{9}$ Shah et al. published a series of 56 children aged from 1 to 10 years who presented either with an untreated clubfoot or recurrent clubfoot. ${ }^{10}$ Ponseti protocols were undertaken, including percutaneous tenotomy of the Achilles tendon. An average of 7.4 casts was required in untreated patients. The relapse rate was $31 \%$, and these children were effectively treated by recasting, bracing, and tibialis anterior tendon transfer (TATT). A supple plantigrade foot was achieved in all cases. Khan and Kumar studied the Ponseti method in 21 children over 7 years of age. ${ }^{11}$ Approximately $85.7 \%$ achieved full correction at a mean follow-up of 4.7 years. The recurrence rate was $24 \%$. A further study in Nepal by Banskota et al. in 36 Nepalese older children between ages 5 and 10 years followed for a minimum of 2 years showed that a plantigrade foot was achieved in $84 \% .{ }^{12}$ The mean number of casts was 9.5. Half of the patients required additional surgery in the form of posterior release, posteromedial release, or osteotomy. It is 
evident, therefore, that it is more difficult to achieve full correction with the Ponseti method of casting as children grow older, and that adjunctive surgery may be necessary.

A systematic review and meta-analysis of observational studies of the Ponseti method in children after walking age showed an average of $89 \%$ having acceptable pain-free plantigrade feet with a recurrence rate of $18 \% .{ }^{13}$ Cumulative experience treating walking-aged children using the principles of Ponseti are, therefore, optimistic. More casts are required, averaging between 6 and 12 . Often there is a lag phase with the first two or three casts showing little improvement. Percutaneous Achilles tenotomy has been proven effective and safe in children of all ages without significant complications (Fig. 2).

\section{A Programmatic Approach to Treating Walking-age Clubfoot in LMICs}

The Ponseti method of treating children born with clubfoot has proven efficacy in $>90 \%$ of babies undergoing the full treatment protocol and is the widely utilized standard of care in developed countries with comprehensive health services. ${ }^{14}$ In LMICs, however, access to skilled providers and adequate health systems is limited. It is estimated that $<15 \%$ of children born with clubfoot deformity in LMICs have access to starting treatment. ${ }^{15}$ Even in countries where a public health approach has been taken, with public-private partnerships developing national programs, it is estimated that coverage averages $<25 \% .{ }^{15}$ The result is a large burden of children worldwide suffering untreated clubfoot deformity.

Studies on the barriers to care in LMIC's consistently point to transportation logistics, lack of financial resources to complete the care, lack of plaster and braces, and lack of available skilled practitioners as causative. ${ }^{16}$ Effective treatment programs need to keep this in mind. Community-based rehabilitation is an effective collaboration in overcoming these barriers. ${ }^{17}$ Developing national programs utilizing public health principles, including task shifting of casting to non-surgeon providers, has improved access and coverage. ${ }^{18}$ Because of transportation logistics, some families benefit from being kept in hospital or rehabilitation step-down units for the duration of the treatment process, in which case an accelerated program of casting twice weekly is effective and well-tolerated, reducing transportation pressures and enabling caregivers an earlier return to their home environments where they are often needed for agrarian work. ${ }^{19}$

\section{Modifications of the Ponseti Technique}

Purists of the Ponseti technique would suggest that Dr Ponseti's protocols should be adhered to strictly with no modification. As a child ages, however, there is a necessity to modify the protocols while staying true to the principles espoused by Ponseti. As such
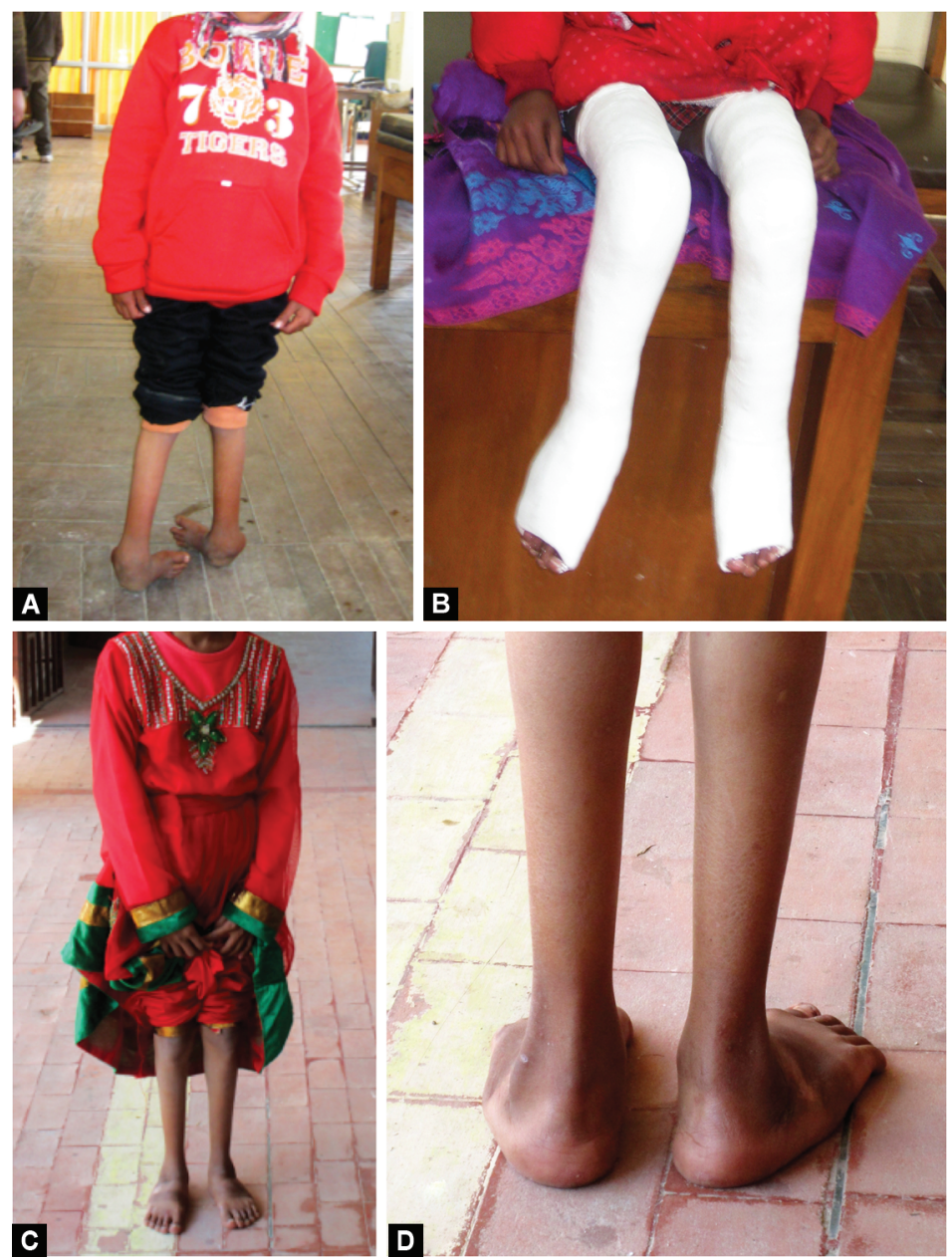

Figs $2 A$ to D: A child presenting at age 6 with untreated clubfoot (A). Treatment using Ponseti principles with casting and Achilles tenotomy (B) with a good result (C and $D)$ 
it would be more accurate to refer to clubfoot treatment in older children as "using the principles of the Ponseti technique".

This is exemplified by a study by Mehtani et al. from India in 41 children presenting after walking age. ${ }^{20}$ They recognized that the Ponseti method needed minor adaptations in this age group. More casts were required (mean 6.9), 94\% of feet achieved painless plantigrade feet without extensive soft tissue surgery, and the recurrence rate was $10.6 \%$. The adaptations necessary were: to continue correcting cavus after the first cast, to continue serial casting after tenotomy to achieve further dorsiflexion, and to utilize below-knee casts after tenotomy and before application of the night-time abduction brace to allow walking.

Significant cavus at presentation is more recalcitrant to treat in the older child, with persistent cavus being one of the recognized outcome problems. Cavus does not automatically correct with the first cast alone as in newborns. Mehtani suggested the correction of the abduction and cavus simultaneously from the first cast. ${ }^{20}$ Where the cavus has not been corrected fully by the time of tenotomy, a simultaneous section of the plantar fascia can be performed. In general, the midfoot corrects more readily than the rear foot with casting. Persistent equinus is the most resistant of the deformities to correct and may remain after tenotomy. In this case, continuing serial casting at weekly intervals allows stretching of the capsular structures, and a further improvement in dorsiflexion can be achieved. This is important to children in cultures where squatting is necessary for many community functions, including toileting. More than plantigrade dorsiflexion is necessary to squat comfortably, and children lacking dorsiflexion beyond neutral have difficulty negotiating the inclines of hilly and mountainous terrains.

The strict Ponseti technique requires the cast to be toe to the groin, with the knee at $90^{\circ}$. Maintaining this position in walking-age children is a significant disability, especially if treatment is bilateral, and experienced practitioners have applied the cast at $45^{\circ}$ of knee flexion to allow some weight-bearing during the treatment process. This is considered enough flexion to be able to control the abduction of the corrected foot.

\section{Bracing Controversies}

An intrinsic part of the Ponseti method of clubfoot care is the bracing protocol implemented after the corrective phase, lasting 3-5 years. A significant barrier to clubfoot care in low-resource environments is the lack of access to appropriate braces and the challenges of adherence to the long-bracing protocol. Local manufacture of low-cost braces using local materials has been found to make bracing more available in the low-income context. ${ }^{21}$

When treatment is implemented after walking age, the question is how much bracing is necessary and for how long? Is it possible to brace a walking child full-time for 3 months following correction? Some practitioners have recommended immediate use of night and nap time bracing up until 5 years of age, bypassing full-time bracing. In children over age 5 at the time of correction, it is not known what the best bracing protocol might be. Some practitioners utilize a period of night bracing. Others utilize an ankle-foot abduction orthosis (AFO) part-time. Protocols for the use of abduction braces after treatment in walking-age children have not been standardized and there is a need for future research.

Ponseti recommended $70^{\circ}$ of foot abduction angle in the brace after treatment. A normal baby's foot easily accomplishes that range, but the abduction range diminishes as a child grows. It has been debated whether a $45^{\circ}$ angle in braces might be satisfactory for walking-age children. A study by Gupta et al., however, showed that the thigh-foot angle is maintained through the growing years. ${ }^{22}$ Moreover, the abduction angle in the brace reflects not just foot abduction, but an external rotation of the hip. It is likely therefore that children of all ages can accommodate to a $70^{\circ}$ abduction angle in the brace. In low-resource settings, utilizing the Steenbeek foot abduction brace and similar locally produced braces, standardizing a $70^{\circ}$ angle bilaterally simplifies manufacturing and avoids confusion among caregivers.

\section{Treatment of Relapse}

In their meta-analysis of studies of walking-age children with clubfoot, Ferreira et al. showed significantly higher rates of relapse in older children than in newborns. ${ }^{13}$ Relapse may be treated in the same manner by repeat serial casting, with or without repeat Achilles tenotomy. Treating the relapse, however, can be more difficult than the primary treatment, requiring longer casting and with a significant failure rate. Since relapse is a significant concern after primary correction, some practitioners have recommended early TATT at the time of tenotomy in older children. Tibialis anterior tendon transfer potentially obviates the need for bracing altogether. In the context of LMICs, with serious logistic barriers to follow-up, early TATT as a prophylactic procedure is seductive. This surgical procedure is efficacious for persistent dynamic supination after correction and for cases of relapse. Ponseti recommended it for children over age 3 years. Since transfer is made into the third cuneiform, it is advised that an ossification center is present to ensure solid fixation. Lang et al., in a study on ossification of the third cuneiform, suggested that the cuneiform is not of appropriate size for transfer until 3 years of age. ${ }^{23}$ Their study suggested earlier transfer would disrupt the enchondral ossification of the cuneiform resulting in deformity. There are anecdotal reports of the transfer of the tendon through the cartilaginous cuneiform but no reported follow-ups. The potential complication of deformity of the third cuneiform is not known but is not likely to be significant.

\section{Adjunctive Surgery in the Older Child}

While extending the use of Ponseti principles to older children has resulted in a paradigm shift in moving the needle towards conservative treatment, surgery is still occasionally indicated in the recalcitrant deformity or with repeated relapses. The surgical principle to be recommended in the treatment of clubfoot deformity is to have a low threshold to perform extra-articular procedures, including percutaneous Achilles tenotomy, plantar fasciotomy, and TATT, with a high threshold for capsular releases and osteotomies.

\section{The Role of Soft Tissue Surgeries}

Most studies have utilized formal posteromedial soft tissue release (PMR) for the failure of manipulation and casting. Complete subtalar release, as formerly practiced with the Turco or Cincinnati techniques, is seldom if ever required. Posteromedial soft tissue release focuses on sectioning of the posterior capsule of the ankle and subtalar joint, potentially followed by repeat serial casting.

\section{Role of Osteotomies}

A plethora of osteotomies have been described for the various foot deformities resulting from previously operated relapsed or 

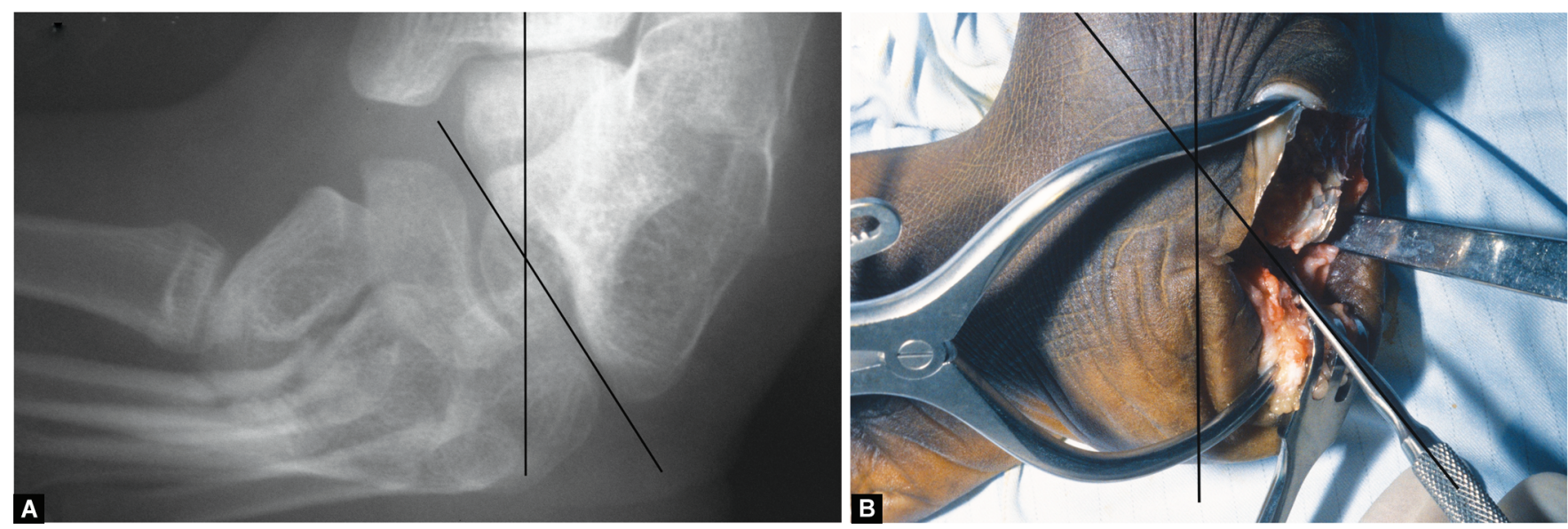

Figs $3 \mathrm{~A}$ and B: (A) Radiograph of an untreated clubfoot in an older child showing obliquity of the calcaneocuboid joint. (B) A surgical case in the same orientation with calcaneocuboid joint exposed and an instrument in the joint demonstrating obliquity with reference to the axis of the leg

recurrent clubfoot deformity, analysis of which is not the focus of this report. For the untreated clubfoot, bony procedures are usually required in the much older child, close to or after skeletal maturity. ${ }^{3,24}$ The exception is the scenario of a surgical outreach to remote underserved communities in the poorest countries where local surgical, rehabilitative, and follow-up resources are in short supply and where there is a lack of trained clubfoot casting practitioners. As noted above, at least half of the children in the world likely live in this circumstance. As such they may have only one opportunity during an outreach mission for correction of a potentially lifelong disabling impairment, in which case primary surgical correction with soft tissue release and/or osteotomy may be their only hope. Surgeons, therefore, need to retain a complement of surgical skills utilized in previous eras. ${ }^{24}$ Since primary soft tissue release alone is accompanied by the potential of significant wound breakdown and relapse, adding osteotomy procedures, notably shortening the lateral column of the foot, reduces the stretch on the medial skin, and arthrodesis reduces the risk of subsequent relapse.

The notable deformity of the tarsal bones seen in older children with clubfoot is the obliquity of the calcaneocuboid joint (Fig. 3). The lateral column of the foot is longer than the medial column. The most useful osteotomy technique, therefore, is shortening of the lateral column (Fig. 4). This can be done either by excision of the anterior process of the calcaneus (Litchblau procedure), calcaneocuboid joint wedge resection (Evans procedure), or cuboid decancellation. ${ }^{3}$ Cuboid decancellation and the Litchblau procedure potentially preserve motion through the articulations while shortening the lateral column but may risk relapse. The Evans-type calcaneocuboid arthrodesis provides stability and potentially reduces the risk of relapse. A long-term study of the Evans procedure for clubfoot done before the Ponseti era showed remarkably good results. ${ }^{25}$

\section{Triple Arthrodesis}

Orthopedic surgeons of a generation ago opined that any serious foot deformity could be corrected by triple arthrodesis. Triple arthrodesis becomes a useful technique in treating much older children or adults with neglected clubfoot deformity. A trial of pre-casting to stretch the medial skin and correct some of the midfoot deformities minimized bone resection. The objectives of arthrodesis are to shorten the lateral column by aggressive resection

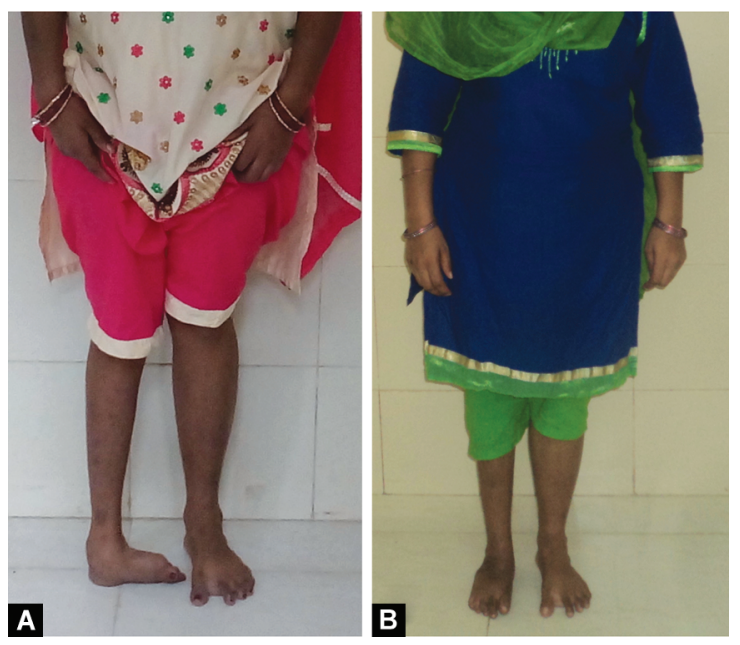

Figs 4A and B: A young woman aged 26 with untreated clubfoot (A). After casting using Ponseti principles, Achilles tenotomy, plantar fasciotomy, and lateral column shortening (B) she has a pain-free plantigrade foot

of bone from the anterior process of the calcaneus, and correct the equinus deformity by utilization of a modified Lambrinudi arthrodesis as described by Penny ${ }^{3}$ (Fig. 5). Once arthrodesis has been accomplished, relapse is unlikely. A long-term study of triple arthrodesis has shown it to be an effective procedure in obtaining a plantigrade workable foot. ${ }^{26}$

\section{External Fixation}

In the pre-Ponseti era, soft tissue distraction with external fixation was utilized with the hope of avoiding extensive soft tissue dissection and release. There was initial enthusiasm to utilize the Ilizarov fixator in neglected or difficult cases (Fig. 6). Experience has been gained in India with the practical and low-cost Joshi (JESS) fixator. ${ }^{27}$ These techniques require attention to detail and specific expertise, often taking many cases over many years to gain competency, and require multiple trips back to the operating room for adjustment and wire replacement. It was Ponseti who pointed out the importance of understanding the kinematics of the tarsal joints with reference to clubfoot deformity, and that the midfoot 

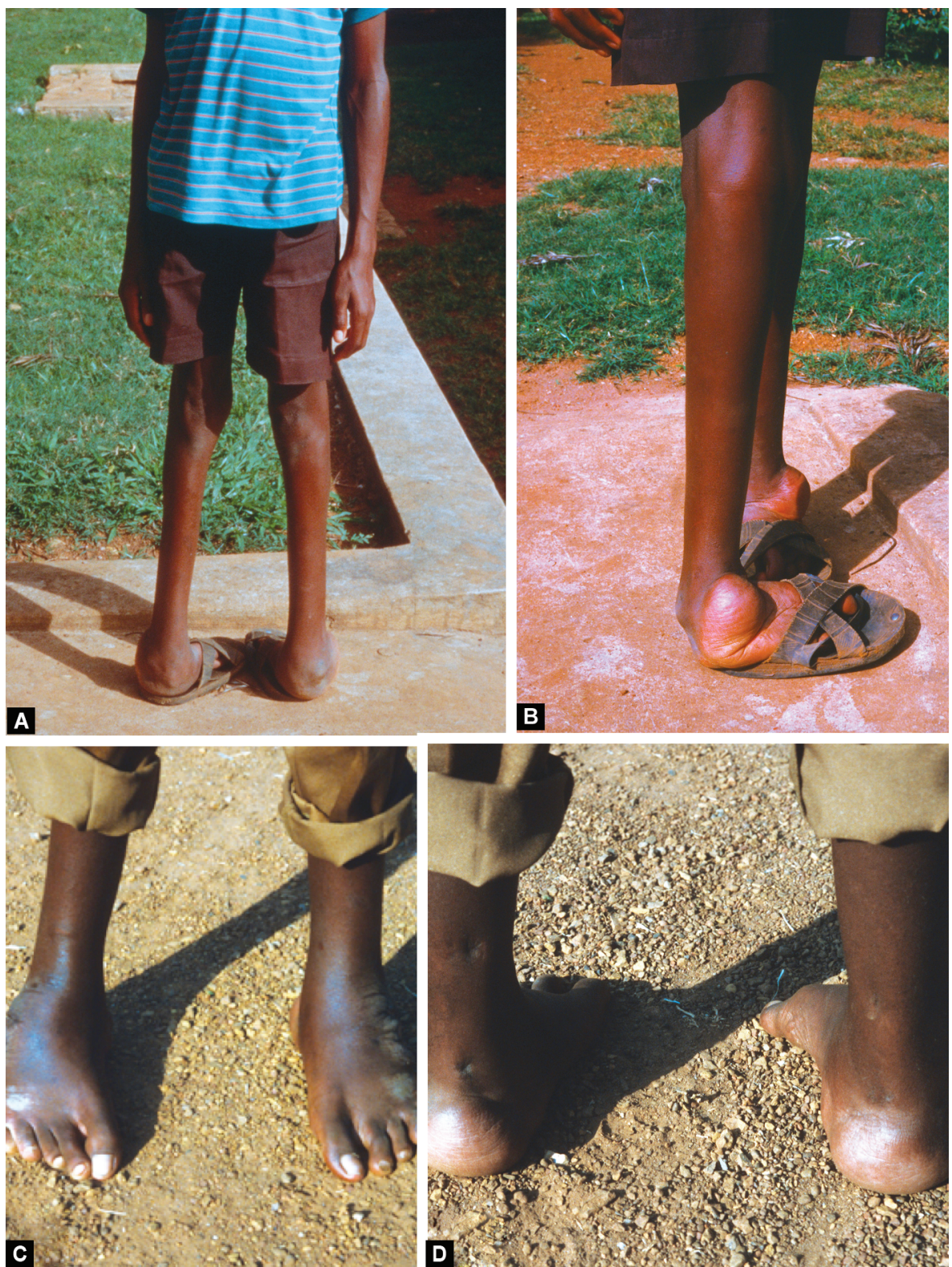

Figs 5A to D: A 14-year-old boy presenting with untreated stiff clubfeet (A and B). After bilateral Lambrinudi triple arthrodesis (C and D) both feet are plantigrade

correction needs to simultaneously correct adduction, inversion, plantarflexion, and heel varus rather than the preexisting Kite concept of correcting these deformities sequentially. This makes for a complex correction in three planes of deformity, something difficult to achieve in the JESS or llizarov fixator.

Hexapod frames, such as the Taylor Spatial Frame, have superseded llizarov type frames, and provide great flexibility and accuracy of correction in all three planes of deformity. The principles of Ponseti correction can be applied by utilizing a fixation wire in the neck of the talus, rotating the subtalar joint around a fixed point. ${ }^{24}$

There has been considerable anecdotal use of external fixators in treating clubfeet, but most published studies constitute limited case studies. While correction of the foot contour is possible, maintaining the correction is difficult without a lengthy time of immobilization in the frame after correction. Complications of stiffness and dysesthetic pain are problematic. A longer-term follow-up study by Freedman et al. showed $>50 \%$ of patients achieved a poor outcome, with associated residual or recurrent deformity often requiring further surgery. ${ }^{28}$ It is likely that the best utilization of external fixation is in cases with very stiff and resistant deformities, such as in cases of arthrogryposis and much older children or adults. External fixation has proven to be a useful technique in relapsed clubfeet after extensive soft tissue surgical dissection when repeat surgical exposure risks vascular compromise. Osteotomy techniques combined with hexapod correction have been described for severe foot deformities after previous surgery. ${ }^{24}$ Tissue distraction can also be used as a preliminary before osteotomy, stretching connective tissues, to minimize bone resection. All such techniques necessarily result in a significantly stiffened foot. 


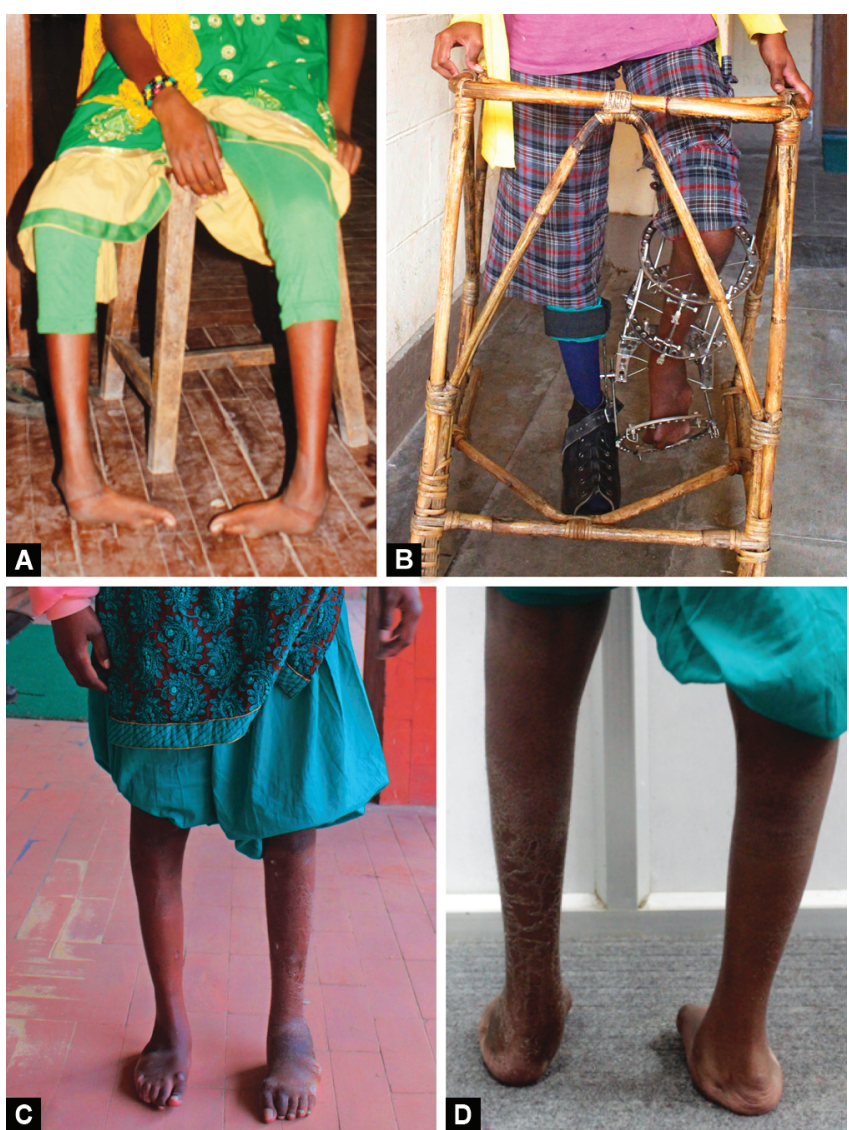

Figs 6 A to D: A 10-year-old child presenting with untreated clubfoot (A) treated with the llizarov external fixator (B) showing a plantigrade foot at follow-up (C and D)

In the scenario of LMICs with the barriers of transportation, housing, and follow-up being significant factors, utilization of external fixation is no more efficient in overcoming treatment barriers than casting using Ponseti principles, while requiring significant surgical expertise, and is therefore difficult to rationalize.

\section{SUMMARY-RECOMMENDATIONS FOR TREATMENT IN 2021}

Wherever it is possible, all children, regardless of age, with untreated clubfoot deformity should undergo manipulation and casting using the principles of the Ponseti method. Longer casting periods can be expected in older children and continued serial casting is often necessary after tenotomy. Casting with the knee in $45^{\circ}$ of flexion allows ambulation in children of walking age and reduces the risk of knee stiffness. Percutaneous tenotomy can safely be done in all age groups. Transfer of the tibialis anterior tendon, once the lateral cuneiform has an adequate ossific center, bypasses the need for prolonged abduction bracing. In much older children, lateral column shortening osteotomy is necessary for complete correction. Modified Lambrinudi triple arthrodesis is a salvage procedure in the older child and young adult when correction cannot be obtained by casting or limited osteotomy and in cases of problematic relapse.

Much progress has been made around the world to implement coordinated clubfoot programs using public health principles, yet a large proportion of children in low-resource environments continue to experience barriers to care resulting in lifelong disabling impairment. Continued effort is needed so that every newborn with clubfoot receives early treatment with the Ponseti method, making more aggressive methods of treatment for late presenting untreated clubfoot deformity unnecessary.

\section{References}

1. Pirani S, Zeznik L, Hodges D. Magnetic resonance imaging study of the congenital clubfoot treated with the Ponseti method. J Pediatr Orthop 2001;21(6):719-726. DOI: 10.1097/01241398-200111000-00004.

2. Nogueira MP, Amaral DT. How much remodeling is possible in a clubfoot treatment? magnetic resonance imaging study in a sevenyear-old child. J Limb Lengthen Reconstr 2018;4(1):49-54. DOI: 10.4103/jllr.jllr_18_17.

3. Penny JN. The neglected clubfoot. Techniq Orthopaed 2005;20(2):153166. DOI: $10.1097 / 01$. bto.0000162987.08300.5e.

4. Nunn TR, Etsub M, Tilahun T, et al. Development and validation of a delayed presenting clubfoot score to predict the response to Ponseti casting for children aged 2-10. Strategies Trauma Limb Reconstr 2018;13(3):171-177. DOI: 10.1007/s11751-018-0324-z.

5. Böhm S, Sinclair MF. The PBS score-a clinical assessment tool for the ambulatory and recurrent clubfoot. J Child Orthop 2019;13(3):282292. DOI: 10.1302/1863-2548.13.190077.

6. Lourenco AF, Morcuende JA. Correction of neglected idiopathic clubfoot by the Ponseti method. J Bone Joint Surg Br 2007;89(3):378381. DOI: 10.1302/0301-620X.89B3.18313.

7. Nogueira MP, Clubfoot treatment: The Ponseti method in Brazil. Presented at the 2nd. International Conference on Birth Defects and Disabilities in the Developing World. Beijing, China. September 11, 2005.

8. Spiegel DA, Shrestha OP, Sitoula P, et al. Ponseti method for untreated idiopathic clubfeet in Nepalese patients from one to six years of age. Clin Orthop Rel Res 2009;467(5):1164-1170. DOI: 10.1007/s11999-0080600-1.

9. Banskota B, Yadav P, Rajbhandari T, et al. Outcomes of the Ponseti method for untreated clubfeet in Nepalese patients seen between the ages of one and five years and followed for at least 10 years. J Bone Joint Surg Am 2018;100(23):2004-2014. DOI: 10.2106/JBJS.18.00445.

10. Shah A, Mehta R, Aroojis A. The Ponseti method of Clubfoot treatment in walking aged children: is it effective? A study of 56 children from 1 to 10 years of age. J Pediatr Orthop B 2019;28(2):159-166. DOI: 10.1097/ BPB.0000000000000562.

11. Khan SA, Kumar A. Ponseti's manipulation in neglected clubfoot in children more than seven years of age: a prospective evaluation of 25 feet with long-term follow-up. J Paediatr Orthop B 2010;19(5):385389. DOI: 10.1097/BPB.0b013e3283387cc8.

12. Banskota B, Banskota AK, Regmi AK, et al. The Ponseti method in the treatment of children with idiopathic clubfoot presenting between five and ten years of age. Bone Joint J 2013;95-B(12):1721-1725. DOI: 10.1302/0301-620X.95B12.32173.

13. Ferreira GF, Stefani KC, de Podesta Haje D, et al. The Ponseti method in children with clubfoot after walking age - systematic review and meta-analysis of observational studies. PLoS ONE 2018;13(11):e0207153. DOI: 10.1371/journal.pone.0207153.

14. Shabtai L, Specht SC, Herzenberg JE. Worldwide spread of the Ponseti method for clubfoot. World J Orthop 2014;5(5):585-590. DOI: 10.5312/ wjo.v5.i5.585.

15. Owen RM, Capper B, Lavy C. Clubfoot treatment in 2015: a global perspective. BMJ Glob Health 2018;3(4):e000852. DOI: 10.1136/bmjgh2018-000852.

16. McElroy $T$, Konde-Lule J, Neema $S$, et al. Understanding the barriers to clubfoot treatment adherence in Uganda: a rapid ethnographic study. Disabil Rehabil 2007;29(11-12):845-855. DOI: 10.1080/09638280701240102.

17. Penny N, Zulianello R, Steenbeek M. Community based rehabilitation and orthopaedic surgery for children with motor impairments in 
an african context. Disabil Rehabil 2007;29(11-12):839-843. DOI: 10.1080/09638280701240052.

18. Owen RM, Penny JN, Mayo A, et al. A collaborative public health approach to clubfoot intervention in 10 low-income and middleincome countries: 2-year outcomes and lessons learnt. J Pediatr Orthop B 2012;21(4):361-365. DOI: 10.1097/BPB.0b013e3283504006.

19. Islam MS, Masood QM, Bashir A, et al. Results of a standard versus an accelerated Ponseti protocol for clubfoot: a prospective randomized study. Clin Orthop Surg 2020;12(1):100-106. DOI: 10.4055/ cios.2020.12.1.100.

20. Mehtani A, Prakash J, Vijay V, et al. Modified Ponseti technique for management of neglected clubfeet. J Pediatr Orthop B 2018;27(1):6166. DOI: $10.1097 /$ BPB. 0000000000000450.

21. Clubfoot: Ponseti Management. Third Edition. Accessed online November 22, 2020. https://storage.googleapis.com/global-helpcdn/2020/07/5e0684b9-help_cfponseti.pdf.

22. Gupta P, Mittal N, Jindal N, et al. A study of normal foot abduction across various age groups in children. Int Orthop 2017;41(11):23652369. DOI: 10.1007/s00264-017-3603-2.

23. Lang PJ, Avoian T, Sangiorgio SN, et al. Quantification of the ossification of the lateral cuneiform in the feet of young children with unilateral congenital talipes equinovarus. Bone Joint J 2017;99B(8):1109-1114. DOI: 10.1302/0301-620X.99B8.BJJ-2016-0999. R2.

24. Eidelman M, Kotlarsky P, Herzenberg JE. Treatment of relapsed, residual and neglected clubfoot: adjunctive surgery. J Child Orthop 2019;13(3):293-303. DOI: 10.1302/1863-2548.13.190079.

25. Graham GP, Dent CM. Dillwyn evans operation for relapsed clubfoot. Long-term results. J Bone Joint Surg Br 1992;74(3):445-448. DOI: 10.1302/0301-620X.74B3.1587899.

26. Saltzman CL, Fehrle MJ, Cooper RR, et al. Triple arthrodesis: twentyfive and forty-four year average follow-up of the same patients. J Bone Joint Surg Am 1999;81(10):1391-1402. DOI: 10.2106/00004623199910000-00004.

27. Suresh S, Ahmed A, Sharma VK. Role of Joshi's external stabilization system fixator in the management of idiopathic clubfoot. $J$ Orthop Surg (Hong Kong) 2003;11(2):194-201. DOI: 10.1177/ 230949900301100216.

28. Freedman JA, Watts H, Otsuka NY. The llizarov method for the treatment of resistant clubfoot: is it an effective solution? J Pediatr Orthop 2006;26(4):432-437. DOI: 10.1097/01.bpo.0000226276. 70706.0e. 